\title{
Utilidad de la videolaringoscopia como predictor de extubación exitosa en pacientes con quemadura por inhalación. Reporte de casos
}

\author{
Utility of videolaringoscopy as a predictor of successful \\ extubation in patients with inhalation burn. Case report
}

Sandra Viviana Amaya-Venegas,, Héctor Julio Meléndez-Flores," Javier Enrique Fajardo-Rivero*

\author{
*Universidad Industrial de Santander. Colombia.
}

\begin{abstract}
RESUMEN. Introducción: La cicatrización y remodelación después de una quemadura por inhalación aumentan el riesgo de obstrucción de la vía aérea, la mayoría de las veces se opta por garantizar la vía aérea mediante traqueostomía. La visualización del estado inicial y la evolución de la vía aérea quemada predicen las repercusiones de la lesión y podrían determinar el éxito de la extubación. La fibrobroncoscopia no permite la evaluación completa de la extensión de las lesiones en la vía aérea superior que suele ser la más afectada. El videolaringoscopio es un instrumento de fácil acceso y lo hemos utilizado para la evaluación inicial y seguimiento de las lesiones, identificando pacientes que pudieran ser extubados. Reporte de casos: Ingresaron tres pacientes a la unidad de cuidados intensivos con quemaduras graves y compromiso de la vía aérea, a quienes se realizó seguimiento semanal con videolaringoscopia como herramienta para identificar a los pacientes que podrían ser extubados. Conclusión: El seguimiento periódico y la evaluación de la progresión de las lesiones con videolaringoscopia permite evaluar la resolución del edema, la reepitelización de las úlceras y la ausencia de pérdida de tejido, que son algunos de los aspectos que derivaron en una extubación exitosa.
\end{abstract}

Palabras clave: Extubación traqueal, quemaduras por inhalación, lesión por inhalación de humo, laringoscopia.

\section{INTRODUCCIÓN}

La presencia de una lesión de la vía aérea por inhalación aumenta significativamente la morbimortalidad de los pacientes quemados, debido a que el edema de las estructuras supraglóticas puede propiciar la oclusión del tracto

Correspondencia:

Dra. Sandra Viviana Amaya-Venegas

Universidad Industrial de Santander, Colombia.

Correo electrónico: sandra_vivi@hotmail.com

Trabajo recibido: 12-XI-2019; aceptado: 08-V-2020.
ABSTRACT. Introduction: Scarring and remodeling after inhalation burn increase the risk of the airway obstruction, therefore most of the time it is decided to guarantee the airway by tracheostomy. The visualization of initial state and evolution of traumatized airway predict the injury repercussions and can determine the success of extubation. Sometimes fibrobronchoscopy does not allow the full extent evaluation of lesions in the upper airway, which is usually the most affected. The video laryngoscope is an easily accessible instrument, we have used for the initial evaluation and follow-up of the lesions, identifying patients who could be extubated. Case report: Three cases of patients who were admitted to the ICU with severe burns and airway involvement received follow up weekly with videolaryngoscopy, as a tool to identify patients who could be extubated. Conclusion: Periodic monitoring and evaluation of the lesions progress with videolaryngoscopy allows to assess the resolution of edema, the re-epithelization of the ulcers, the absence of tissue loss are some of the aspects that result in a successful extubation.

Keywords: Smoke inhalation injury, inhalation burns, airway extubation, laryngoscopy.

respiratorio y generar asfixia entre otras complicaciones. La intubación temprana es prioritaria en pacientes con sospecha de quemadura por inhalación ante la inminente progresión y pérdida de los reparos anatómicos. ${ }^{1}$

Existen recomendaciones para el manejo de las lesiones por quemadura en la vía aérea; sin embargo, no se han establecido criterios de extubación de acuerdo con el compromiso en la vía aérea debido a la variabilidad de la inflamación, cicatrización y subsecuente remodelación de las áreas comprometidas. ${ }^{2}$

Contar con herramientas que aporten información objetiva para definir el manejo definitivo de la vía aérea tendría efectos en los tiempos de estancia en la unidad de cuidados intensivos $(\mathrm{UCl})$, costos y complicaciones. 


\section{DESCRIPCIÓN DE LOS CASOS}

El día 26 de marzo se presentó una afluencia masiva de quemados expuestos a llamas directas en recinto cerrado, sufriendo quemaduras graves, de estos pacientes algunos fallecen por la extensión y compromiso de las quemaduras al llegar a urgencias. Se internaron en la $\mathrm{UCI}$ tres pacientes con quemaduras graves con compromiso facial y de áreas especiales que requirieron lavado y desbridamiento. En el abordaje de la vía aérea por parte del anestesiólogo se documentaron lesiones en la vía aérea con restos carbonosos y edema de las estructuras supraglóticas, se manejaron con intubación orotraqueal y se trasladaron intubados a la UCI.

Se trata de tres adolescentes sin patologías previas con antecedente de consumo de sustancias psicoactivas, lo que se vio reflejado en una alta demanda de opioides y sedantes durante su estancia en la $\mathrm{UCl}$ y, posteriormente, síndrome de abstinencia que dificultó el destete de la sedación (Tabla 1).

Durante su estancia requirieron soporte vasopresor y reanimación hídrica por inestabilidad hemodinámica, se fijaron metas hemodinámicas y de perfusión con adecuada respuesta.

Inicialmente sin alteraciones del parénquima pulmonar en la radiografía de tórax, no se administró corticoide ni se realizó manejo específico para la lesión en vía área salvo medidas generales como hidratación, ventilación mecánica con parámetros protectores e higiene bronquial. Presentaron compromiso infeccioso asociado con la ventilación requiriendo manejo antibiótico específico guiado por cultivo y antibiograma, sólo hubo compromiso sistémico en el paciente más comprometido por las quemaduras que requirió manejo específico. Una vez resueltas las complicaciones se planteó la liberación de la ventilación mecánica, se realizó seguimiento de las lesiones de la vía aérea mediante videolaringoscopia directa con el dispositivo C - Mac (Figura 1).

El caso I mostró resolución del edema, reepitelización de las úlceras, ausencia de pérdida de tejido y resolución o ausencia de lesiones sobre las cuerdas vocales, criterios con los que se definió la extubación orotraqueal, después de un destete ventilatorio difícil por episodios de abstinencia, previo test de fuga positivo, se logró la extubación exitosa después de 18 días de ventilación mecánica invasiva (VMI) y al paciente se le dio egreso de la $\mathrm{UCl}$ a las 72 horas de mantener un patrón espontáneo sin complicaciones.

El caso II presentó el compromiso más importante en cuanto a la extensión de la quemadura así como de la severidad de las lesiones en vía aérea, este paciente persistió con ulceraciones y edema de las estructuras supraglóticas incluso tres semanas después de la exposición al fuego, por lo que se definió realización de traqueostomía percutánea y se logró el destete de la ventilación mecánica a las 72 horas.

Tabla 1: Resumen de casos que muestra las características clínicas más relevantes y la evolución de los tres casos.

\begin{tabular}{|c|c|c|c|}
\hline & Casol & Caso II & Caso III \\
\hline Edad (años) & 17 & 17 & 17 \\
\hline Quemadura en SCT (\%) & 35 & 55 & 22 \\
\hline Área comprometida & $\begin{array}{l}\text { Cara, cuello, tórax y espalda } \\
\text { superior, hombros y antebrazos } \\
\text { bilateral }\end{array}$ & $\begin{array}{l}\text { Cabeza, pabellón auricular, cuello, } \\
\text { tronco, M. sup. bilateral, muslo y } \\
\text { rodilla izquierda }\end{array}$ & $\begin{array}{l}\text { Cabeza, cuello, tórax anterior, M. } \\
\text { sup. bilateral y muslos bilaterales }\end{array}$ \\
\hline Quemadura de vía aérea & Sí & Sí & Sí \\
\hline Antecedentes & Consumo de SPA & Consumo de SPA & Consumo de SPA \\
\hline Soporte vasopresor & Sí & Sí & Sí \\
\hline Compromiso sistémico & No & $\begin{array}{l}\text { Falla renal AKI II sin TRR } \\
\text { Edema agudo de pulmón }\end{array}$ & No \\
\hline Infeccioso & $\begin{array}{l}\text { CSB: Enterobacter } \\
\text { cloacae sensible }\end{array}$ & $\begin{array}{l}\text { CSB: Pseudomonas } \\
\text { aeruginosa MDR }\end{array}$ & $\begin{array}{c}\text { CSB: Klebsiella } \\
\text { pneumoniae sensible }\end{array}$ \\
\hline $\begin{array}{l}\text { Tiempo de intubación } \\
\text { (días) }\end{array}$ & $\begin{array}{c}18 \\
\text { Extubación }\end{array}$ & $\begin{array}{c}17 \\
\text { Traqueostomía }\end{array}$ & $\begin{array}{c}17 \\
\text { Traqueostomía }\end{array}$ \\
\hline Días de VMI & 18 & 20 & 19 \\
\hline Días de UCl & 21 & 26 & 21 \\
\hline
\end{tabular}

SCT = superficie corporal total; $\mathrm{M}$. sup. = miembros superiores; $\mathrm{SPA}=$ sustancias psicoactiva, $\mathrm{AKI}$ = lesión renal aguda; $\mathrm{TRR}=$ terapia de reemplazo renal; $\mathrm{CSB}=\mathrm{cultivo}$ de secreción bronquial; $\mathrm{VMI}$ = ventilación mecánica invasiva; $\mathrm{UCI}$ = Unidad de Cuidados Intensivos. 

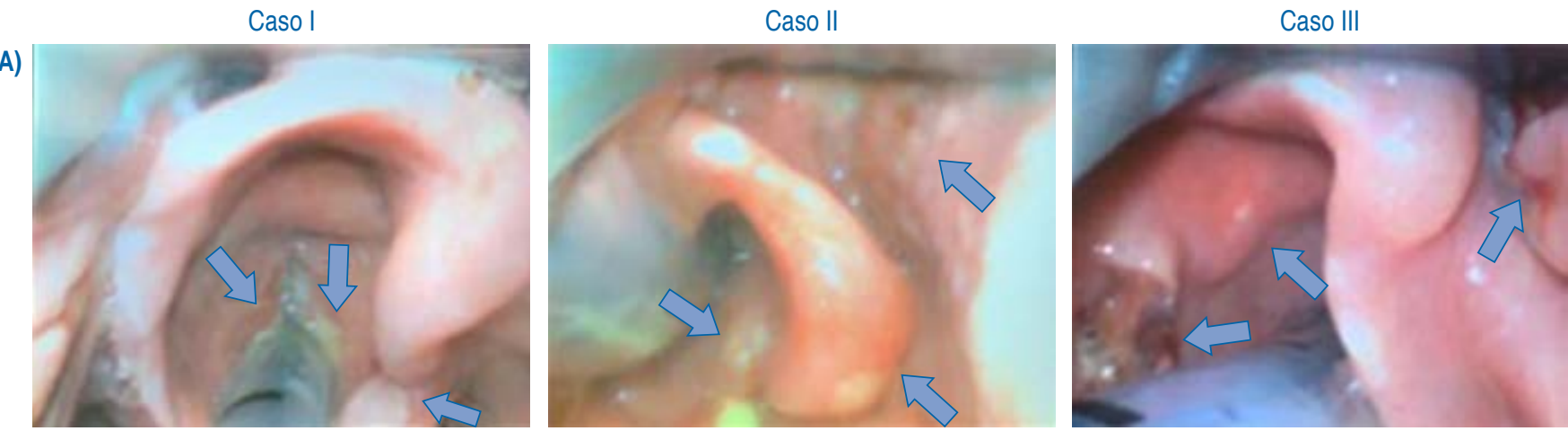

B)
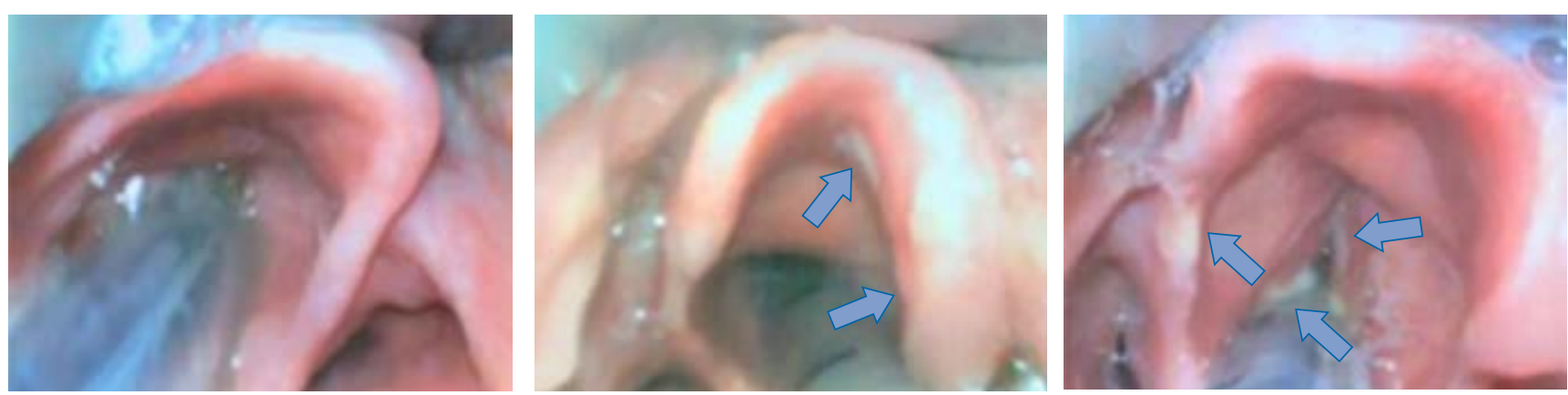

Figura 1: Hallazgos en las videolaringoscopias. A) Primeras fotos. Se observan úlceras y componente inflamatorio y erosivo a causa de inhalación de vapores (día 1); B) Fotos de seguimiento. Se aprecian las lesiones en proceso de resolución (día 5).

El caso III evidenció persistencia del edema de las estructuras supraglóticas después de tres semanas de la exposición al fuego, además de una lesión importante con necrosis de cartílago epiglótico y del surco aritenoepiglótico izquierdo, el cual presenta riesgo de formación de cicatrices. El manejo del caso con traqueostomía percutánea se define en conjunto con el cirujano de cabeza y cuello. Se logró la liberación de la ventilación mecánica 48 horas después y se le dio egreso a piso tras tolerar 48 horas en tienda de traqueostomía con patrón espontáneo.

\section{DISCUSIÓN}

La presencia de una lesión por inhalación aumenta significativamente la morbimortalidad asociada con las lesiones por quemadura. Los mecanismos de lesión de la vía aérea incluyen quemadura directa por inhalación de gases calientes, lesiones químicas en la tráquea y los bronquios, deterioro de los procesos de transporte y utilización de oxígeno por inhalación de monóxido de carbono y cianuro. ${ }^{1}$

El diagnóstico se basa en la historia de exposición al fuego y al humo, en especial cuando hay pérdida de conciencia o atrapamiento en un espacio cerrado. Al examen físico las quemaduras en la cara, nariz, vello nasal o facial, esputo carbonoso, hipoxia, voz ronca, broncorrea o broncoespasmo hacen sospechar de lesión; la visualización de residuos carbonosos, eritema o ulceración a la laringoscopia confirman el diagnóstico.

Las radiografías de tórax generalmente son normales, el daño al parénquima pulmonar no es el resultado de una lesión térmica directa, es causado por los productos incompletos de la combustión y el desarrollo de complicaciones como inflamación, infección o atelectasia, lo que provoca desajustes en la ventilación, alteración del intercambio de gases, la distensibilidad pulmonar y la perfusión. Sólo dos tercios de los pacientes desarrollan cambios difusos, opacidades focales o edema pulmonar de cinco a 10 días después de la lesión. ${ }^{2}$

Las lesiones en la vía respiratoria pueden no ser evidentes inicialmente, pero con la resucitación masiva con líquido pueden generar edema en estructuras supraglóticas con prolapso de tejidos que pueden ocluir la vía aérea y generar asfixia e intubación difícil, por lo que ante la sospecha de lesión se indica la intubación temprana. ${ }^{3}$

La puntuación abreviada de lesiones (AIS, del inglés abbreviated injury scale) asigna un puntaje de gravedad de 0 (sin lesión) a cuatro (lesión masiva), según los hallazgos iniciales, las lesiones varían desde una descamación superficial leve, edema de mucosa, ulceraciones, necrosis focal y pérdida de tejido. Los grados superiores de lesión se han asociado con una oxigenación más deficiente y mayor duración de la ventilación mecánica; sin embargo, no se correlaciona con un aumento de la 
mortalidad. Con el tiempo las lesiones pueden resolver o progresar a la formación de sinequias, traqueomalacias, ulceraciones o granulomas secundaria a la cicatrización y remodelación anómala, deformando o produciendo obstrucción respiratoria. ${ }^{4}$

Las víctimas de la lesión por inhalación pueden desarrollar síndrome de dificultad respiratoria, complicaciones como traqueobronquitis, bronquiectasias, bronquiolitis y neumonía (38-60\%). ${ }^{5}$

Las recomendaciones para el manejo de la vía aérea quemada incluyen la intubación temprana, la elevación de la cabecera a $30^{\circ}$, evitar la reanimación hídrica excesiva, la humidificación del oxígeno administrado, la higiene bronquial, los parámetros protectores de ventilación mecánica y la terapia farmacológica con broncodilatadores y micronebulizaciones. ${ }^{2}$

No hay una recomendación sobre el manejo a largo plazo de estos pacientes, ni directrices que determinen cuándo un paciente con quemadura de la vía aérea podría ser extubado o cuándo se beneficie de la traqueostomía. Definir rápidamente el manejo definitivo de la vía aérea podría acortar el tiempo de estancia en la UCl.

El seguimiento de las lesiones con videolaringoscopia permite tener un registro de las lesiones presentes y evaluar su progresión y su tendencia con el tiempo. La resolución del edema y la reepitelización de las úlceras, la ausencia de pérdida de tejido y la ausencia de lesiones sobre las cuerdas vocales se utilizaron como guía para identificar al paciente susceptible de extubación; asimismo, la presencia de alguno de estos hallazgos determinó la realización de traqueostomía para el mantenimiento de la vía aérea.

\section{CONCLUSIONES}

Visualizar el estado de la vía aérea superior mediante laringoscopia directa permite hacernos una idea de la gravedad de las lesiones por inhalación, la posibilidad del seguimiento periódico y la documentación de la EVOLUCIÓN de las lesiones hace de la videolaringoscopia una herramienta útil para identificar pacientes con quemadura de la vía aérea que podrían ser extubados facilitando su rápido egreso de la UCI.

\section{REFERENCIAS}

1. Bittner EA, Shank E, Woodson L, Martyn JA. Acute and perioperative care of the burn-injured patient. Anesthesiology 2015;122(2):448-464. https://doi.org/10.1097/aln.0000000000000559

2. Mlcak RP, Suman OE, Herndon DN. Respiratory management of inhalation injury. Elsevier Burns 2007;33(1):2-13. https://doi.org/10.1016/j. burns.2006.07.007

3. Rehberg S, Maybauer MO, Enkhbaatar P, Maybauer DM, Yamamoto Y, Traber DL. Pathophysiology, management and treatment of smoke inhalation injury. Expert Rev Respir Med 2019;3(3):283-297. https:// doi.org/10.1586/ers.09.21

4. Jones SW, Williams FN, Cairns BA, Cartotto R. Inhalation injury: pathophysiology, diagnosis, and treatment. Clin Plast Surg 2017;44(3):505-511. https://doi.org/10.1016/j.cps.2017.02.009

5. Gupta K, Mehrotra M, Kumar P, Gogia AR, Prasad A, Fisher JA. Smoke inhalation injury: etiopathogenesis, diagnosis, and management. Indian J Crit Care Med 2018;22(3):180-188. https://doi.org/10.4103/ ijccm.ijccm_460_17

Conflicto de intereses: Los autores declaran no tener conflicto de intereses. 